\title{
Soluble receptor and gene polymorphism for AGE: relationship with obesity and cardiovascular risks
}

\author{
Mohamed Rowisha', Manal El-Batch², Thanaa El Shikh², Salwa El Melegy ${ }^{2}$ and Hany Aly ${ }^{3}$
}

BACKGROUND: Obesity in adolescents has quadrupled in the past $30 \mathrm{y}$. Markers for cardiovascular risks are needed in this population. We hypothesized that soluble receptor for advanced glycation end products (sRAGE) and asymmetric dimethyl arginine (ADMA) can correlate with carotid intimamedia thickness (CIMT), a known index of subclinical atherosclerosis. We also aimed to evaluate the frequency of (Gly82Ser) RAGE gene polymorphism in obese adolescents.

METHODS: Obese and nonobese adolescents were evaluated in a cross-sectional study for lipid profile, insulin resistance, ADMA, sRAGE, and RAGE gene (Gly 82 Ser) polymorphism. We measured cIMT in all subjects and performed correlation analyses with all markers.

RESULTS: The study included 50 obese and 40 healthy control adolescents. Compared to controls, obese subjects had less SRAGE $(P=0.02)$ and greater CIMT $(P=0.006)$, insulin resistance $(P<0.0001)$, and ADMA $(P<0.0001)$. In a multivariate linear regression model, sRAGE was associated with cIMT ( $\beta=0.28$, $P=0.04)$. Both GS and SS genotypes of RAGE were more frequent in obese than controls $(P=0.04)$.

CONCLUSION: Increased ADMA and decreased SRAGE are associated with cardiovascular risks in obese adolescents. The $S$ allele in RAGE gene is more frequently detected with obesity. The role of RAGE gene and mechanisms leading to cardiovascular risks need further studying.

C hildhood obesity is an emerging public health challenge with an estimated prevalence of around $20 \%$ in western countries (1). Exposure to cardiovascular risk factors in early life, including obesity, may induce changes in the arteries that are associated with the diagnosis of atherosclerosis in adults (2). The measurement of carotid intimamedia thickness (cIMT) is a noninvasive test to detect early alterations in arterial wall and can be a useful screening tool to early assess subclinical manifestations of cardiovascular and metabolic diseases (3). However, there is a desperate need to discover novel biomarkers that can help understand the exact pathophysiology and improve clinical management of cardiovascular diseases associated with obesity in children (4).
Asymmetric dimethyl arginine (ADMA) is a competitive inhibitor of endothelial nitric oxide (NO) synthase that reduces the production of $\mathrm{NO}$ and therefore might cause endothelial dysfunction. Serum ADMA concentrations are increased in individuals with hypercholesterolemia, atherosclerosis, hypertension, chronic heart failure, diabetes mellitus, and chronic renal failure. It has been suggested as a potential risk predictor for cardiovascular events and all-cause cardiovascular mortality in patients with coronary artery disease (5). The relationship between ADMA and cardiovascular complications in children remains unclear.

The advanced glycation end products (AGE) and its receptor (RAGE) system is a newly discovered pathway implicated in the pathogenesis of several cardio-metabolic diseases (6). The RAGE is a multiligand receptor of the immunoglobulin superfamily of cell surface molecules and engages diverse ligands, including the AGEs. sRAGE, a scavenger that prevents ligand binding to RAGE, is a soluble receptor produced by alternative splicing of RAGE messenger RNA (7). These soluble forms may act as a naturally occurring inhibitor of the signaling induced by the interaction of AGEs with its cellular receptor. Also, it may neutralize AGE-mediated damage by acting as a decoy (8). Polymorphisms in the RAGE gene may alter AGE processing in tissues or reactions after the binding of AGEs to RAGE. Of specific interest is the RAGE G82S polymorphism; because of its location in the ligand-binding $\mathrm{V}$ domain of RAGE in which substitution of Gly (82) with Ser that promotes glycosylation of RAGE at asp81; it is associated with enhanced inflammatory responses, increased ligand binding, downstream signaling as well as RAGE expression (9).

In this cross-sectional study, we aimed to investigate the presence of subclinical atherosclerosis in obese and nonobese adolescents using cIMT. We measured two novel biomarkers, ADMA and sRAGE, as predictors of cIMT. We also tested the association of polymorphism in RAGE gene with obesity in adolescents. To our understanding, this is the first study to correlate these biomarkers with cIMT in childhood obesity.

\section{RESULTS}

We studied 90 subjects; of them 50 were obese and 40 had normal BMI. There was no difference in age between the obese and

\footnotetext{
'Department of Pediatrics, Faculty of Medicine, Tanta University, Tanta, Egypt; ${ }^{2}$ Department of Medical Biochemistry, Faculty of Medicine, Tanta University, Tanta, Egypt: ${ }^{3}$ Department of Pediatrics, the George Washington University and Children's National Medical Center, Washington, DC. Correspondence: Hany Aly (haly@mfa.gwu.edu) Received 3 October 2015; accepted 30 December 2015; advance online publication 13 April 2016. doi:10.1038/pr.2016.55
} 


\section{Articles $\mid$ Rowishaetal.}

the control groups. When compared to the control group, the obese group had significantly less concentration of HDL-C and greater concentrations for all other values in the lipid profile and insulin resistance (HOMA-IR). The clinical and laboratory characteristics of the studied groups are presented in Table $\mathbf{1}$. cIMT was significantly increased in the obese group. The obese group had increased expression of plasma ADMA and AGE, whereas the plasma concentration of sRAGE was decreased (Figure 1). cIMT correlated significantly with the three studied biomarkers ADMA, AGA, and sRAGE. It correlated with

Table 1. Clinical characteristics and laboratory values for the study population $(n=90)$

\begin{tabular}{lccc}
\hline & $\begin{array}{c}\text { Control } \\
(n=40)\end{array}$ & $\begin{array}{c}\text { Obese } \\
(n=50)\end{array}$ & $P$ \\
\hline Age (years) & $13.8 \pm 1.9$ & $13.7 \pm 2.1$ & 0.82 \\
BMI $\left(\mathrm{kg} / \mathrm{m}^{2}\right)$ & $19.3 \pm 1.9$ & $27.5 \pm 4.1$ & $<0.0001$ \\
Waist-hip ratio & $0.80 \pm 0.04$ & $0.85 \pm 0.03$ & $<0.0001$ \\
Systolic blood pressure $(\mathrm{mmHg})$ & $98 \pm 8$ & $115 \pm 15$ & $<0.0001$ \\
Diastolic blood pressure $(\mathrm{mmHg})$ & $65 \pm 6$ & $85 \pm 12$ & $<0.0001$ \\
Fasting blood glucose $(\mathrm{mg} / \mathrm{dl})$ & $97.1 \pm 4.5$ & $99.2 \pm 6.1$ & 0.07 \\
Triglycerides $(\mathrm{mg} / \mathrm{dl})$ & $130.1 \pm 8.3$ & $149.8 \pm 9.5$ & $<0.0001$ \\
Cholesterol, total (mg/dl) & $165.3 \pm 9.3$ & $181.1 \pm 10.5$ & $<0.0001$ \\
Cholesterol, LDL $(\mathrm{mg} / \mathrm{dl})$ & $83.7 \pm 7.4$ & $105.6 \pm 9.8$ & $<0.0001$ \\
Cholesterol, HDL $(\mathrm{mg} / \mathrm{dl})$ & $54.9 \pm 5.4$ & $44.5 \pm 4.9$ & $<0.0001$ \\
Plasma insulin $(\mu \mathrm{lU} / \mathrm{ml})$ & $7.3 \pm 2.5$ & $12.4 \pm 3.2$ & $<0.0001$ \\
Insulin resistance $(\mathrm{HOMA}-\mathrm{IR})$ & $1.7 \pm 0.5$ & $2.9 \pm 1.2$ & $<0.0001$ \\
\hline
\end{tabular}

Data are expressed in mean \pm SD.

$\mathrm{HDL}$, high-density lipoprotein cholesterol; HOMA-IR, homeostatic model assessment of insulin resistance; $L D L$, low-density lipoprotein.

a

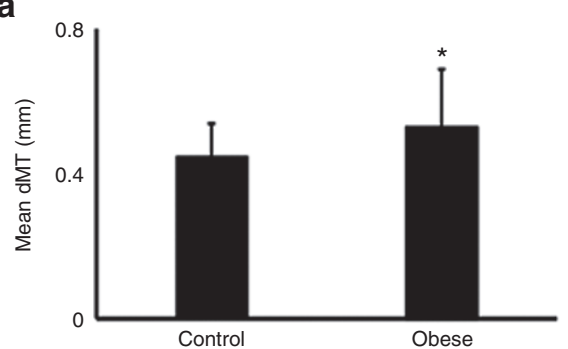

C

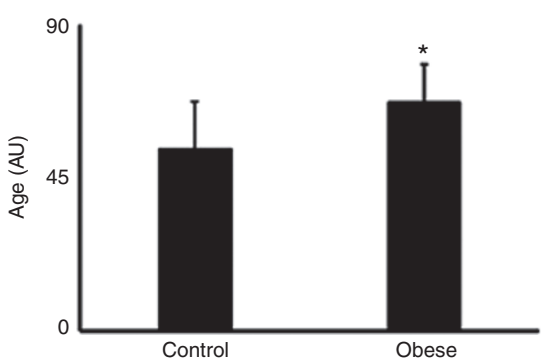

BMI, and insulin resistance; however, it did not correlate with subjects' age or blood pressure (Table 2).

The relationships between the three studied biomarkers and metabolic measures in obese subjects $(n=50)$ were performed using Pearson's correlation analysis. ADMA correlated significantly with HDL $(r=-0.43, P=0.002)$; AGE correlated with insulin resistance $(r=0.286, P=0.04)$; and sRAGE correlated with LDL ( $r=0.35, P=0.012)$. sRAGE was the only marker to correlate with BMI $(r=0.29, P=0.04)$. All three markers significantly correlated with CIMT $(r=0.42, P=0.003, r=$ $0.31, P=0.027$, and $r=0.45, P=0.001$, respectively).

A multivariate linear regression model was structured with cIMT being the dependent variable. Out of the three studied markers, only sRAGE was significantly associated with cIMT $(\beta=0.28, P=0.04)$. Other independent variables that were significant include age $(\beta=0.32, P=0.02)$, BMI $(\beta=0.35, P=$ $0.005)$, and $\operatorname{HDL}(\beta=-0.28, P=0.03)$.

Figure 2 demonstrates the genotyping of RAGE gene (Gly 82 Ser) polymorphism (GG, GS, and SS). The rare homozygous (SS) and the heterozygous (GS) mutants for G82S polymorphisms were represented more frequently in the obese group than controls (odds ratios $(\mathrm{OR})=3.9,95 \%$ confidence intervals $(\mathrm{CI})=1.2-12.8 ; P=0.04)$. The $\mathrm{S}$ allele was more frequently observed in obese $(\mathrm{OR}=4.5,95 \% \mathrm{CI}=1.9-17.2 ; P=0.002)$. Obese adolescents, when compared to other genotypes, those with SS genotype had significantly increased HOMA-IR, AGE, and ADMA and decreased sRAGE (Table 3).

\section{DISCUSSION}

We demonstrated the increased expression of ADMA and AGE and decreased expression of sRAGE in the plasma of obese adolescents; cIMT, as a surrogate to cardiovascular complications, increased significantly in the obese group when

b

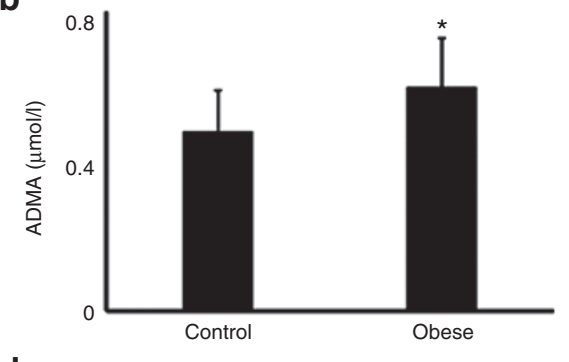

d

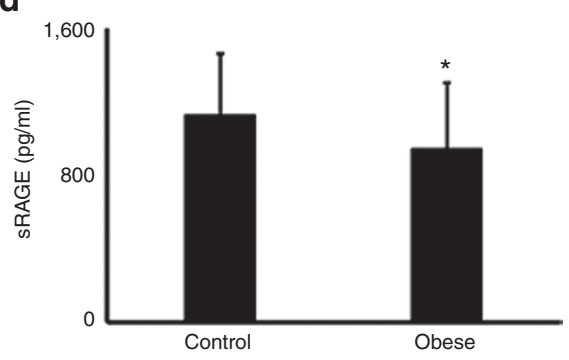

Figure 1. Comparisons between obese and control groups in CIMT, ADMA, AGE, and SRAGE. ADMA, asymmetric dimethyl arginine; AGE, advanced glycation end products; cIMT, carotid intima-media thickness; SRAGE, soluble receptor for advanced glycation end products. Panel a demonstrates cIMT to be significantly greater in obese subjects than controls $(P=-0.06)$. Panel $\mathbf{b}$ demonstrates ADMA to be significantly greater in obese subjects than controls $(P<0.0001)$. Panel $\mathbf{c}$ demonstrates AGE to be significantly greater in obese subjects than controls $(P=-0.0001)$. Panel $\mathbf{d}$ demonstrates sRAGE to be significantly smaller in obese subjects than controls $(P=0.02)$. 
compared to controls. This is the first study to show association between AGE gene (Gly 82 Ser) polymorphism and obesity. The Ser82Ser (SS) and the Gly82Ser (GS) genotypes, and the Ser82 (S) allele frequency were significantly greater in obese adolescents than controls. Within the obese group, the subgroup with SS genotype had significantly decreased plasma

Table 2. Pearson's correlation analysis between carotid intima-media thickness and vascular risk factors in the obese group $(n=50)$

\begin{tabular}{lcc}
\hline Variables & $\begin{array}{c}\text { Correlation } \\
\text { coefficient }\end{array}$ & $P$ value \\
\hline Age (years) & 0.15 & 0.28 \\
BMI $\left(\mathrm{kg} / \mathrm{m}^{2}\right)$ & 0.38 & 0.006 \\
Systolic blood pressure $(\mathrm{mmHg})$ & 0.18 & 0.22 \\
Diastolic blood pressure $(\mathrm{mmHg})$ & -0.12 & 0.42 \\
Insulin resistance (HOMA-IR) & 0.3 & 0.038 \\
ADMA ( $\mu \mathrm{mol} / \mathrm{l})$ & 0.42 & 0.003 \\
AGE) $/ \mathrm{ml})$ & 0.31 & 0.027 \\
SRAGE $(\mathrm{pg} / \mathrm{ml})$ & 0.45 & 0.001 \\
\hline
\end{tabular}

ADMA, asymmetric dimethyl arginine; AGE, advanced glycation end products; HOMA-IR, homeostatic model assessment of insulin resistance; sRAGE, soluble receptor for advanced glycation end products.

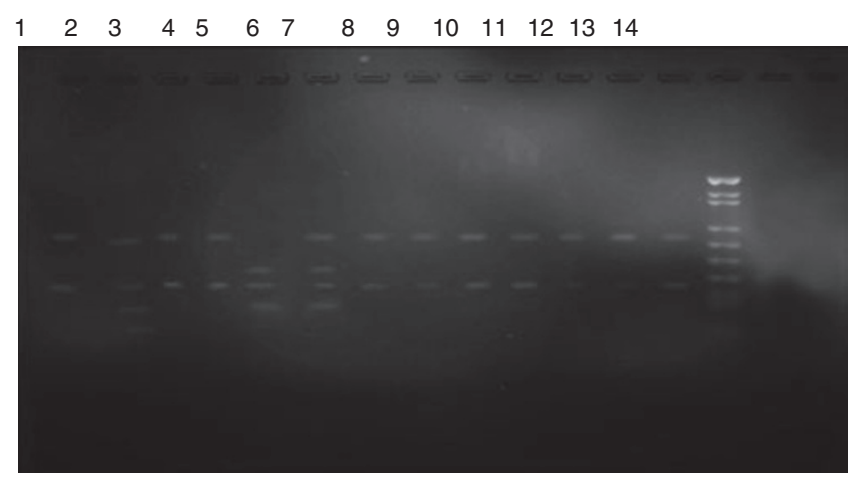

Figure 2. Polymerase chain reaction and restriction fragment length polymorphism analysis of RAGE glycine 82 serine gene polymorphism with Alul separated on an agrose gel. Lane 14 represents $10 \mathrm{bp}$ marker ladder (ultra low range DNA ladder) (34-501 bp) served as reference for DNA fragment; Lanes $\mathbf{1 , 3}, \mathbf{4}, \mathbf{7 , 8} \mathbf{8}, \mathbf{1 0}, \mathbf{1 1}, \mathbf{1 2 , 1 3}$ show 2 bands at 149 and $248 \mathrm{bp}$ and they are homozygous for the absence of new restriction site (GG); Lane 5 shows 3 bands at 67, 149, and $181 \mathrm{bp}$ and it is homozygous for the presence of new restriction site (SS); Lanes $2, \mathbf{6}$ show four bands at $67,149,181$, and $248 \mathrm{bp}$ and it is heterozygous for the absence and presence of a new restriction site (GS). concentration of sRAGE, whereas SBP, DBP, cIMT, triglycerides, insulin resistance, AGE, and ADMA were all increased when compared to Sg and GG obese adolescents.

Increased plasma ADMA concentrations were associated with hypertension, insulin resistance, and increased cIMT in this current study. Obesity is known to impose oxidative stress that can downgrade the activity of dimethyl arginine dimethyl amino hydrolase, the enzyme that metabolizes ADMA, thereby permitting ADMA to accumulate. The mechanism for ADMA to cause cardiovascular risks is via the inhibition endothelial NO that will interfere with endothelial function and vascular structure (10).

We demonstrated increased plasma concentration of AGE in obese adolescents that was associated with increased cIMT, insulin resistance, and hypertension. Circulating AGEs has been recently shown to represent a novel progressive risk marker for predicting possible vascular complications in type II diabetes mellitus (11). The increase in AGE in obese individuals may be related to the presence of chronic inflammation, enhanced oxidative stress, and lower insulin sensitivity (12). A previous study reported decreased AGE in obese children while another did not find a difference between obese and lean groups $(13,14)$. The discrepancy among these reports may be due to the differences in subject characteristics including age and lifestyle. Under pathological conditions, AGE gradually accumulates and exerts its toxicity indirectly via interaction with specific cell surface receptors including the receptor for AGEs (RAGE); that is considered proinflammatory and proatherogenic (15).

In the current study, sRAGE was significantly decreased in obese adolescent as compared to controls. RAGE ligands have better access to RAGE, the binding of which leads to oxidative stress generation and activation of inflammatory pathways (16). As a result of such binding, it has been postulated that the soluble isoform (sRAGE) could play an antagonistic role by their ability to bind toxic AGE, neutralizing their action thus preventing the adverse effects of RAGE signaling (17). We showed obese individuals to have decreased sRAGE; therefore they were deprived from its protective effect. Insulin resistance negatively correlated with sRAGE in our population that might indicate its role in the reduction of RAGE, probably by activating common intracellular signaling pathways (18); however, the cross-sectional design of this study does not allow establishing a cause and effect relationship. The negative association we have described between insulin resistance and sRAGE could be just a response to a modifier that we did not

Table 3. RAGE gene (glycine 82 serine) polymorphism and vascular risk factors in obese adolescents $(n=50)$

\begin{tabular}{|c|c|c|c|c|}
\hline & $\mathrm{GG}(n=35)$ & $\mathrm{GS}(n=7)$ & $\mathrm{SS}(n=8)$ & $P$ value \\
\hline Mean cIMT (mm) & $0.48 \pm 0.09$ & $0.52 \pm 0.11$ & $0.58 \pm 0.13$ & 0.04 \\
\hline Insulin resistance (HOMA-IR) & $2.3 \pm 0.3$ & $2.5 \pm 0.4$ & $3.9 \pm 0.2$ & $<0.0001$ \\
\hline AGE)AU) ${ }^{b}$ & $67(50-80)$ & $74(60-80)$ & $77(57-80)$ & 0.02 \\
\hline sRAGE (pg/ml) & $1,250 \pm 330$ & $920 \pm 320$ & $680 \pm 290$ & $<0.0001$ \\
\hline
\end{tabular}

Data are expressed in mean \pm SD, except ${ }^{a, b}$ data are expressed in median (range). ANOVA test is used except ${ }^{a, b}$ Kruskal-Wallis test is used.

ADMA, asymmetric dimethyl arginine; AGE, advanced glycation end products; cIMT, carotid intima-media thickness; HOMA-IR, homeostatic model assessment of insulin resistance;

sRAGE, soluble receptor for advanced glycation end products. 


\section{Articles $\mid$ Rowishae tal.}

measure. Of note, decreased circulating sRAGE has been independently associated with cardiovascular diseases suggesting its vital role in the pathogenesis of atherosclerosis (19).

In this study, the Ser82Ser (SS) and the Gly82Ser (GS) genotypes and the Ser82 (S) allele frequency were significantly higher in obese adolescent than controls. Obese adolescents with SS genotype had increased blood pressure, insulin resistance, triglycerides, and ADMA, but decreased sRAGE when compared with obese individuals with other genotypes. Ser82 allele was previously associated with microvascular complications in patients with type 2 diabetes (20). How RAGE signaling induces microvascular complications has not been fully elucidated, but this process could have been mediated through the enhanced expression of NF- $\kappa \mathrm{B}$ and, the proinflammatory and profibrotic cytokine, TGF- $\beta$ (21).

Of note, RAGE polymorphisms can determine the individual expression of sRAGE by alteration of the N-glycosylation state of the protein that makes RAGE more vulnerable for the action of proteinases (22).

One of the limitations of this study is that we did not account for physical activity and quality of diet in regression analysis; we did not have objective tools to provide accurate estimates for these two factors in the country of Egypt. The study was conducted exclusively in Egypt. Therefore the findings, especially those related to gene polymorphism, cannot be extrapolated to other populations. We did not longitudinally follow study subjects over the years to detect if and when they develop cardiovascular complications. However, the use of cIMT as a surrogate for cardiovascular risks has been validated in multiple previous studies.

Increased expression of circulating ADMA and AGE but decreased sRAGE in addition to the $S$ allele in the RAGE gene were associated with vascular complications in obese adolescents. The genetic variants Gly82Ser was increased in obese adolescents and could be a used as a marker for vascular complications. Further studies are needed to examine the impact of modifying lifestyle in adolescents with Gly82Ser variants on prevention of obesity-associated vascular complications.

\section{METHODS}

\section{Patients}

This cross-sectional study has been approved by the Institutional Review Board (IRB) at Tanta University Medical Center. Children were informed with the nature and purpose of the study. Parental consents were obtained for all subjects before enrollment. We studied two groups of adolescents with age ranged from 12 to $15 \mathrm{y}$; Obese Group with a body mass index (BMI) of was $\geq 24 \mathrm{~kg} / \mathrm{m}^{2}$ and Control Group with $\mathrm{BMI}<24 \mathrm{~kg} / \mathrm{m}^{2}$. Z-scores for BMI were determined using age and sexspecific cut off points as described by the International Obesity Task Force (23). Subjects were excluded from the study if they had any of the following conditions: (i) diagnosed with any hormonal or genetic causes for obesity, such as growth hormone deficiency, growth hormone resistance, hypothyroidism, leptin deficiency or resistance to leptin action, Cushing syndrome, precocious puberty, polycystic ovary syndrome, prolactin-secreting tumors, Laurence-Moon-Biedl syndrome, Cohen syndrome, Down syndrome, and Turner syndrome; (ii) diagnosed with cardiovascular disorders such as hypertension, coronary artery disease, and peripheral arterial occlusive disease; iii) diagnosed with type-1 diabetes mellitus; iv) used any medications that could affect insulin resistance, lipid profile, cIMT, ADMA, AGE or sRAGE, such as anabolic-androgenic steroids or psychotropics, statins and other lipid-lowering medications, immunosuppressive therapy, prolonged use of NSAID, corticosteroids, hormonal medications, antioxidants, and vitamin supplements; and (v) had a family history of early cardiovascular disease or diabetes. In addition to a complete history and a physical examination, anthropometric measurements were obtained for all subjects including: (i) body weight, measured in light indoor clothes; (ii) height, measured without shoes using a measuring stick; (iii) waist circumferences, measured twice with the subject standing and wearing only underwear using a nonelastic tape at a point midway between the last palpable rib and the iliac crest in a horizontal plane on one visit, and the average of the two measurements was recorded (24); (iv) hip circumference, measured at the widest part at the gluteal region; (v) waist-hip ratio, calculated from previous measurements; and (vi) BMI, calculated as body weight divided by square height $\left(\mathrm{kg} / \mathrm{m}^{2}\right)$.

\section{Blood Pressure Measurement}

Systolic blood pressure and diastolic blood pressure were measured twice at the right arm after a 10-min rest in the supine position by auscultation with mercury sphygmomanometer (Erka, Bad Tölz, Germany). The average of the two measurements was used in the analysis. The sizes of inflatable bladder used were $9 \times 16,13 \times 23$, and $15 \times 30 \mathrm{~cm}^{2}$ according to the individual's arm circumference. Blood pressure was evaluated according to age, gender, and height using currently recommended normative tables (25). Hypertension was determined by reference values of BP percentiles for gender, according to age and height percentile (26).

\section{CIMT}

cIMT was measured by B-mode ultrasound using an instrument equipped with a 4.0-10.0 MHz vascular transducer (Vivid 7; GE Medical Systems, Piscataway, NJ). It was measured by the same experienced radiologist who was blinded to the participant's risk factors and laboratory values. The subjects were examined supine with the neck extended and the probe in the antero-lateral position. The protocol involved repeated manual measurements of the right and left common carotid far wall at $2 \mathrm{~cm}$ proximal to the bulb bifurcation. On a longitudinal B-mode image, the far wall of the common carotid artery appears as two bright parallel lines separated by a hypoechoic space. The distance between the leading edge of the first bright line on the far wall (lumen-intima interface) and the leading edge of the second bright line (media-adventitia interface) indicates the cIMT of the far wall (27). Three measurements of the left and right common carotid were taken and the mean of the greatest measurement of each side represented the cIMT in this study as previously described (3). The intraobserver relative SDs of cIMT measurements was $4.9 \%$ as assessed in a group of 20 subjects examined in our laboratory.

\section{Biochemical Assays}

A total of $5 \mathrm{ml}$ venous blood was obtained from all subjects after an over-night fast for $12 \mathrm{~h}$ and was placed in ethylenediaminetetraacetic acid vacutainer tube (Belliver Industrial Estate, Plymouth, UK) for genetic study. Samples were centrifuged (at 4,000 g for $10 \mathrm{~min}$ at $4{ }^{\circ} \mathrm{C}$ ) and the separated plasma was transferred into plastic tubes and kept at $-80{ }^{\circ} \mathrm{C}$ until further analysis of: (i) fasting blood glucose concentration, (ii) total cholesterol (TC), triglyceride (TG), and high-density lipoprotein-cholesterol (HDL-C) concentrations using standard enzymatic methods (Boehringer Diagnostica and Wako Chemicals, Neuss, Germany). Low-density lipoprotein-cholesterol (LDL-C) concentrations were calculated using Friedewald equation: LDL-C = TC-(HDL-C+ (TG/5)) (28), (iii) insulin, by enzyme-linked immunosorbent assay using ELISA kit (R\&D Systems, Minneapolis). Homoeostasis model (HOMA-IR) for assessment of insulin resistance (IR) was done using the equation: HOMA-IR = (fasting plasma insulin $(\mu \mathrm{IU} / \mathrm{ml}) \times$ fasting plasma glucose $(\mathrm{mg} / \mathrm{dl}) / 405($ ref. $(29))$.

\section{Plasma Concentration of ADMA}

Plasma samples were analyzed using ELISA kit (Immundiagnostik AG, Bensheim, Germany) (30). The Columbus microplate stripwasher (Tecan Trading AG, Switzerland) and Sunrise absorbance reader (Magellan software, Tecan, Männedorf, Switzerland) were utilized. Standards and controls provided with the kits were used in measurements. 


\section{Plasma Concentrations of AGE, sRAGE, and RAGE (Gly 82 Ser) Gene Polymorphism}

AGE was measured using fluorescence spectrophotometric assay method (31). Plasma concentration of sRAGE was determined using a commercial ELISA kit (Aviscera Bioscience, Santa Clara, CA). RAGE (Gly 82 Ser) gene polymorphism was assessed using polymerase chain reaction (PCR) and restriction fragment length polymorphism (RFLP) (32). Briefly, genomic DNA was prepared using Bio spin whole blood genomic DNA extraction kit (Qiagen, Valencia, CA). The extracted DNA was subjected to PCR amplification for RAGE gene using Dream Taq Green PCR Master Mix which includes nuclease-free water and PCR Master Mix twice, in addition to primers (Biolegio, Nijmegen, the Netherlands): the foreword Primer sequence 5'-GTAAGGGGGGCTCCTGTTGCA-3' and the reverse primer sequence 5'-GGCCAAGGCTGGGGTTGAAGG-3'. PCR was carried out into DNA thermal cycler (Biometra, Whatman, Germany) and the amplification program was as follows: initial precycling denaturation for $5 \mathrm{~min}$ at $95^{\circ} \mathrm{C}, 30$ cycles of denaturation at $95^{\circ} \mathrm{C}$ for $1 \mathrm{~min}$, annealing at $58{ }^{\circ} \mathrm{C}$ for $1 \mathrm{~min}$ and extension at $72^{\circ} \mathrm{C}$ for $1 \mathrm{~min}$. This was followed by final extension period at $72{ }^{\circ} \mathrm{C}$ for $10 \mathrm{~min}$. The 397 PCR product was digested using the restriction endonuclease, Fast Digest Alu I enzyme (Thermo Scientific, Pittsburg, PA) at $37^{\circ} \mathrm{C}$ for $16 \mathrm{~h}$ yielding $248+149 \mathrm{bp}$ fragments for wild-type allele (Gly82) and $181+67+149$ bp fragments for mutant allele (Ser82). The restricted products were separated by $3 \%$ agarose gel electrophoresis and visualized on UV trans-illuminator using ethidium bromide staining.

\section{Statistical Analysis}

Statistical analysis was performed using SPSS version 10 (Chicago, IL). Data were expressed as mean $( \pm \mathrm{SD})$ for continuous parametric variables, as median (range) for continuous nonparametric variables and as numbers (\%) for categorical variables. Student's $t$-test was applied to compare quantitative variables between the two studied groups. Oneway ANOVA and Kruskal-Wallis test were used for subgroup analyses of continuous parametric and nonparametric variables, respectively. The frequencies of various alleles and genotypes were compared by $\chi^{2}$ test; OR and 95\% CI were calculated. Pearson's correlation analysis was used to examine the relationships between cIMT and tested vascular risk factors. Associations between cIMT and other risk factors were evaluated by simple regression analysis, followed by multivariate linear regression with forward stepwise addition. All statistical tests were twotailed and only a $P$ value $\leq 0.05$ was considered statistically significant.

\section{ACKNOWLEDGMENTS}

We thank Timour Moustafa at the department of cardiology in the University of Tanta for his performance of cIMT in our study. We thank Veronica Amaya at the George Washington University for her assistance in editing the manuscript and the figures.

Disclosure: No financial assistance was received to support this study. No financial ties to products in the study or potential/perceived conflicts of interest.

\section{REFERENCES}

1. D’Adamo E, Guardamagna O, Chiarelli F, et al. Atherogenic dyslipidemia and cardiovascular risk factors in obese children. Int J Endocrinol 2015;2015:912047.

2. Simşek E, Balta H, Balta Z, Dallar Y. Childhood obesity-related cardiovascular risk factors and carotid intima-media thickness. Turk J Pediatr 2010;52:602-11.

3. Sidhu PS, Desai SR. A simple and reproducible method for assessing intimal-medial thickness of the common carotid artery. Br J Radiol 1997;70:85-9.

4. Nadeau KJ, Maahs DM, Daniels SR, Eckel RH. Childhood obesity and cardiovascular disease: links and prevention strategies. Nat Rev Cardiol 2011;8:513-25.

5. Sibal L, Agarwal SC, Home PD, Boger RH. The role of asymmetric dimethylarginine (ADMA) in endothelial dysfunction and cardiovascular disease. Curr Cardiol Rev 2010;6:82-90.

6. D'Adamo E, Giannini C, Chiavaroli V, et al. What is the significance of soluble and endogenous secretory receptor for advanced glycation end products in liver steatosis in obese prepubertal children? Antioxid Redox Signal 2011;14:1167-72.
7. Kalea AZ, Schmidt AM, Hudson BI. Alternative splicing of RAGE: roles in biology and disease. Front Biosci (Landmark Ed) 2011;16:2756-70.

8. Vazzana N, Santilli F, Cuccurullo C, Davì G. Soluble forms of RAGE in internal medicine. Intern Emerg Med 2009;4:389-401.

9. Park SJ, Kleffmann T, Hessian PA. The G82S polymorphism promotes glycosylation of the receptor for advanced glycation end products (RAGE) at asparagine 81: comparison of wild-type rage with the G82S polymorphic variant. J Biol Chem 2011;286:21384-92.

10. Mauricio MD, Aldasoro M, Ortega J, Vila JM. Endothelial dysfunction in morbid obesity. Curr Pharm Des 2013;19:5718-29.

11. Bansal S, Chawla D, Siddarth M, Banerjee BD, Madhu SV, Tripathi AK. A study on serum advanced glycation end products and its association with oxidative stress and paraoxonase activity in type 2 diabetic patients with vascular complications. Clin Biochem 2013;46:109-14.

12. Amin MN, Mosa AA, El-Shishtawy MM. Clinical study of advanced glycation end products in egyptian diabetic obese and non-obese patients. Int $\mathrm{J}$ Biomed Sci 2011;7:191-200.

13. Sebeková K, Somoza V, Jarcusková M, Heidland A, Podracká L. Plasma advanced glycation end products are decreased in obese children compared with lean controls. Int J Pediatr Obes 2009;4:112-8.

14. Kim OY, Jo SH, Jang Y, et al. G allele at RAGE SNP82 is associated with proinflammatory markers in obese subjects. Nutr Res 2009;29:106-13.

15. Kovacic P, Somanathan R. Cell signaling and receptors in toxicity of advanced glycation end products (AGEs): $\alpha$-dicarbonyls, radicals, oxidative stress and antioxidants. J Recept Signal Transduct Res 2011;31:332-9.

16. Daffu G, del Pozo CH, O’Shea KM, Ananthakrishnan R, Ramasamy R, Schmidt AM. Radical roles for RAGE in the pathogenesis of oxidative stress in cardiovascular diseases and beyond. Int J Mol Sci 2013;14:19891-910.

17. Fukami K, Yamagishi S, Okuda S. Role of AGEs-RAGE system in cardiovascular disease. Curr Pharm Des 2014;20:2395-402.

18. Unoki H, Yamagishi S. Advanced glycation end products and insulin resistance. Curr Pharm Des 2008;14:987-9.

19. Falcone C, Bozzini S, D’Angelo A, et al. Plasma levels of soluble receptor for advanced glycation end products and coronary atherosclerosis: possible correlation with clinical presentation. Dis Markers 2013;35:135-40.

20. Tripathi AK, Chawla D, Bansal S, Banerjee BD, Madhu SV, Kalra OP. Association of RAGE gene polymorphism with vascular complications in Indian type 2 diabetes mellitus patients. Diabetes Res Clin Pract 2014;103:474-81.

21. Xie J, Méndez JD, Méndez-Valenzuela V, Aguilar-Hernández MM. Cellular signalling of the receptor for advanced glycation end products (RAGE). Cell Signal 2013;25:2185-97.

22. Osawa M, Yamamoto Y, Munesue S, et al. De-N-glycosylation or G82S mutation of RAGE sensitizes its interaction with advanced glycation endproducts. Biochim Biophys Acta 2007;1770:1468-74.

23. Cole TJ, Bellizzi MC, Flegal KM, Dietz WH. Establishing a standard definition for child overweight and obesity worldwide: international survey. BMJ 2000;320:1240-3.

24. McCarthy HD, Jarrett KV, Crawley HF. The development of waist circumference percentiles in British children aged 5.0-16.9 y. Eur J Clin Nutr 2001;55:902-7.

25. National High Blood Pressure Education Program Working Group on High Blood Pressure in Children and Adolescents. The fourth report on the diagnosis, evaluation, and treatment of high blood pressure in children and adolescents. Pediatrics 2004;114:555-76.

26. Redwine KM, Falkner B. Progression of prehypertension to hypertension in adolescents. Curr Hypertens Rep 2012;14:619-25.

27. Guardamagna O, Abello F, Saracco P, Baracco V, Rolfo E, Pirro M. Endothelial activation, inflammation and premature atherosclerosis in children with familial dyslipidemia. Atherosclerosis 2009;207:471-5.

28. Friedewald WT, Levy RI, Fredrickson DS. Estimation of the concentration of low-density lipoprotein cholesterol in plasma, without use of the preparative ultracentrifuge. Clin Chem 1972;18:499-502.

29. Duseja A, Thumburu KK, Das A, et al. Insulin tolerance test is comparable to homeostasis model assessment for insulin resistance in patients with nonalcoholic fatty liver disease. Indian J Gastroenterol 2007;26:170-3.

30. Schulze F, Wesemann R, Schwedhelm E, et al. Determination of asymmetric dimethylarginine (ADMA) using a novel ELISA assay. Clin Chem Lab Med 2004;42:1377-83.

31. Kalousová M, Zima T, Malbohan IM, Stípek S. [Determination of advanced glycation end products]. Sb Lek 2002;103:427-34.

32. Gu H, Yang L, Sun Q, et al. Gly82Ser polymorphism of the receptor for advanced glycation end products is associated with an increased risk of gastric cancer in a Chinese population. Clin Cancer Res 2008;14:3627-32. 